\title{
On the Performance of CP based Exponentially Weighted Block RLS Channel Estimation Algorithm for OFDM Systems
}

\author{
Hassan Ali* \\ Faculty of Engineering and Built Environment \\ The University of Newcastle, Australia \\ Singapore Campus \\ Hassan.Ali@newcastle.edu.au
}

\author{
Pooh Ling E \\ School of Electrical and Electronic Engineering \\ University of Nottingham \\ Malaysia Campus \\ eyx6ple@nottingham.edu.my
}

\begin{abstract}
Cyclic Prefix (CP) based block Recursive Least Squares $(R L S)$ channel estimation algorithms have been proposed for Orthogonal Frequency Division Multiplexing (OFDM) systems. In this paper, we investigate the performance of $C P$ based exponentially weighted block RLS channel estimator. Our analysis and extensive simulation results show that smaller values of exponential forgetting weightings cause increase in convergence time and steady state performance error of the algorithm. Furthermore, performance of the approach degrades with the increase in channel nulls and constellation size.
\end{abstract}

\section{Introduction}

Orthogonal frequency division multiplexing (OFDM) has received growing interest recently for its use in wireless and wireline communication systems [6, 1, 2, 3]. OFDM systems are based on the insertion of the so-called cyclic prefix $(\mathrm{CP})$ which consists of redundant symbols replicated at the beginning of each transmitted block. The $\mathrm{CP}$ insertion and the inverse FFT (IFFT) operation at the transmitter not only eliminate inter-block-interference (IBI) between successive blocks, but also enable simple one tap equalization in the frequency domain. At the receiver, after $\mathrm{CP}$ removal and FFT processing of each data block, transmitted symbols are recovered through one tap equalization operation (to compensate for the channel amplitude and phase shifts). Hence, for coherent detection knowledge of channel impulse response is very important. As the channel impulse response is not known at the receiver, it needs to be estimated efficiently.

${ }^{*}$ The author gratefully acknowledges the support of this work by The University of Newcastle, Australia under Grant No. G0189378.
In applications such as xDSL [3], channel is estimated through some initial training process and retraining is required to track the channel variation. To avoid the system overhead due to retraining and thus to estimate the channel more efficiently, in [4], a block recursive leastsquares (RLS) channel estimation algorithm exploiting the CP present in the OFDM system is proposed. The approach relies on the simple one tap minimum-mean-square-error (MMSE) equalization to estimate the transmitted CP part. In [5], the scheme is reformulated as CP based exponentially weighted block RLS filtering scheme and by replacing the one-tap MMSE equalization with the simple onetap zero forcing (ZF) equalization. Xiaowen and Liu in [5], analyzed the performance of the scheme considering both channel noise and decision error and showed that the algorithm is guaranteed to converge with proper loading.

In this paper, we take a closer look and investigate the performance of the scheme by varying exponential forgetting weightings, channel nulls and modulation size. It is found that the decrease in exponential forgetting weightings not only contribute to the increase in convergence time of the algorithm but also degradation in its steady state performance. Moreover, performance of the approach degrades with the increase in channel nulls and constellation size.

\section{CP based Exponentially Weighted Block Adaptive Channel Estimation Algorithm}

In this section, we review the CP-OFDM system and CP based exponentially weighted adaptive channel estimation algorithm $[5]^{1}$.

Fig. 1 depicts the baseband discrete time block equivalent model of a CP-OFDM system with adaptive channel estimation. The system has $m / 2$ complex parallel subchannels. The input data are buffered to blocks, each data block

\footnotetext{
${ }^{1}$ For consistency, we closely follow the notation in $[4,5]$.
} 

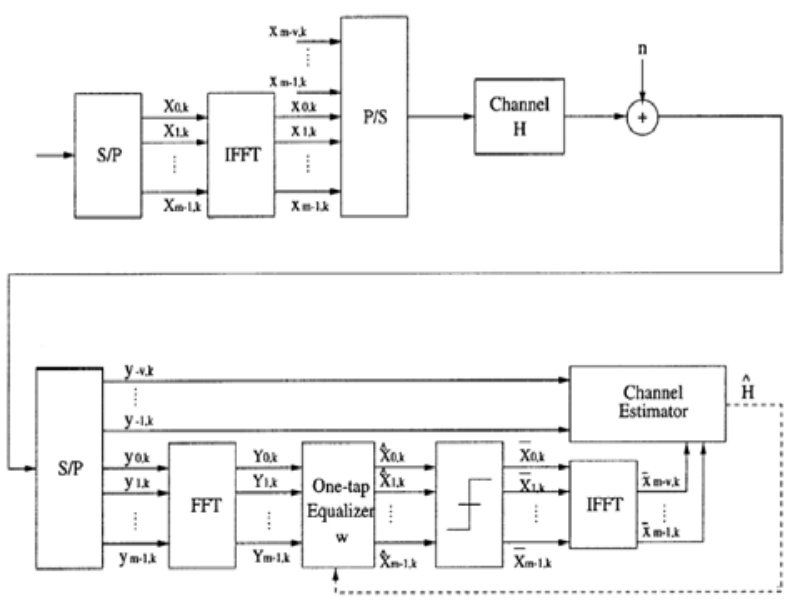

Figure 1. The CP-OFDM system with adaptive channel estimation [5].

is divided into $m / 2$ bit streams and then mapped to complex constellation points $X_{i, k}, i=0, \ldots, m / 2-1$ at time $k$. After $m$-point IFFT on $\mathbf{X}_{k}=\left[X_{0, k}, X_{1, k}, \ldots, X_{m-1, k}\right]^{T}$ (here the last $m / 2$ samples are just the conjugates of the first $m / 2$ samples), the modulated time domain signal is $\mathbf{x}_{k}=\left[x_{0, k}, x_{1, k}, \cdots, x_{m-1, k}\right]^{T}$.

A CP $\mathbf{x}_{k}^{(f)}=\left[x_{-v, k}, \cdots, x_{-1, k}\right]^{T}$ (where $x_{-i, k}=$ $x_{m-i, k}$ and $\left.i=1, \ldots, v\right)$ is then appended in front of $\mathbf{x}_{k}$ before transmission through the channel having impulse response $\mathbf{h}=\left[h_{0}, h_{1}, \cdots, h_{v}\right]^{T}$. At the receiver, the prefix part $\mathbf{y}_{k}^{(f)}=\left[y_{-v, k}, \cdots, y_{-1, k}\right]^{T}$ is removed.

The relationship between prefix part $\mathbf{y}_{k}^{(f)}$ and the transmitted signal can be expressed as

$$
\mathbf{y}_{k}^{(f)}=\mathbf{A}_{k} \mathbf{h}_{k}+\mathbf{n}_{k}^{(f)}
$$

where

$\mathbf{A}_{k}=\left[\begin{array}{c}\mathbf{u}_{v, k}^{H} \\ \vdots \\ \mathbf{u}_{2, k}^{H} \\ \mathbf{u}_{1, k}^{H}\end{array}\right]=\left[\begin{array}{cccc}x_{-v, k} & x_{m-1, k-1} & \cdots & x_{m-v, k-1} \\ \ddots & \ddots & \ddots & \vdots \\ \ddots & \ddots & \ddots & \vdots \\ x_{-1, k} & \cdots & x_{-v, k} & x_{m-1, k-1}\end{array}\right]$, $\mathbf{n}_{k}^{(f)}=\left[n_{-v, k}, \cdots, n_{-1, k}\right]^{T}$ and $n_{i, k} \sim \mathcal{N}\left(0, \sigma^{2}\right)$ is the channel noise.

After the FFT operation on $\mathbf{y}_{k}=$ $\left[y_{0, k}, y_{1, k}, \cdots, y_{m-1, k}\right]^{T}$, the demodulated signal is $\mathbf{Y}_{k}=\left[Y_{0, k}, Y_{1, k}, \cdots, Y_{m-1, k}\right]^{T}$.

The $\mathrm{CP}$ removes IBI between $\mathbf{X}_{k}$ 's and we can thus write

$$
Y_{i, k}=X_{i, k} \mathcal{H}_{i}+N_{i, k}, \quad i=0, \ldots, m-1,
$$

where $\mathcal{H}_{i}=\sum_{l=0}^{v} h_{l} e^{-j((2 \pi i l) / m)}$ is the channel frequency response and $N_{i, k}=(1 / \sqrt{m}) \sum_{l=0}^{m-1} n_{l, k} e^{-j((2 \pi l i) / m)} \sim$ $\mathcal{N}\left(0, \sigma^{2}\right)$ is the noise of the $i$ th subchannel.
According to (2), only standard one-tap equalizer $W_{i}$ is needed to get the estimation of $X_{i, k}$ from $Y_{i, k}$, i.e., $\hat{X}_{i, k}=$ $Y_{i, k} W_{i}$. The $\mathrm{ZF}$ one-tap equalizer for the $i$ th subchannel is given by $W_{i}=1 / \mathcal{H}_{i}$. The decision is then made on $\hat{X}_{i, k}$ to get the final output $\bar{X}_{i, k}=q\left(\hat{X}_{i, k}\right)$, where $q(\cdot)$ is the decision operation.

Based on the relationship (1), an exponentially weighted block RLS algorithm is adopted to adaptively estimate the channel. First, the estimated transmitted $\mathrm{CP}$ is obtained by performing FFT on the decision-directed samples $\bar{X}_{i, k}$ 's, which are obtained through ZF equalization. The estimated correlation matrix $\overline{\boldsymbol{\Phi}}_{k}$ and the correlation vector $\overline{\mathbf{z}}_{k}$ are formed as:

$$
\begin{aligned}
\overline{\mathbf{\Phi}}_{k} & =\mu_{1} \overline{\mathbf{\Phi}}_{k-1}+\mathbf{A}_{k}^{H} \Lambda_{v} \mathbf{A}_{k} \\
& =\mu_{1} \overline{\mathbf{\Phi}}_{k-1}+\sum_{l=1}^{v} \mu_{2}^{l-1} \overline{\mathbf{u}}_{l, k} \overline{\mathbf{u}}_{l, k}^{H}, \\
\overline{\mathbf{z}}_{k} & =\mu_{1} \overline{\mathbf{z}}_{k-1}+\mathbf{A}_{k}^{H} \Lambda_{v} \mathbf{y}_{k}^{(f)} \\
& =\mu_{1} \overline{\mathbf{z}}_{k-1}+\sum_{l=1}^{v} \mu_{2}^{l-1} \overline{\mathbf{u}}_{l, k} y_{-l, k},
\end{aligned}
$$

where the exponential weighting diagonal matrix $\Lambda_{v}=\operatorname{diag}\left\{\mu_{2}^{v-1}, \ldots, \mu_{2}, 1\right\}$ (carries exponential forgetting weightings on its diagonal); and $\mu_{1}$ and $\mu_{2}$ are forgetting factors across blocks and within blocks, respectively. The factors $\mu_{1}$ and $\mu_{2}$ are both assumed equal or less than one. The estimated data vector $\overline{\mathbf{u}}_{l, k}$ is formed as $\overline{\mathbf{u}}_{l, k}=\left[\bar{x}_{-l, k}, \ldots, \bar{x}_{-v, k}, \bar{x}_{-1, k-1}, \ldots, \bar{x}_{-l, k-1}\right]^{T}$, $l=v, v-1, \ldots, 1$. The channel estimation is then obtained as $\hat{\mathbf{h}}_{k}=\overline{\mathbf{\Phi}}_{k}^{-1} \overline{\mathbf{z}}_{k}$.

Based on the above discussion, CP based exponentially weighted block RLS algorithm is summarized in Table 1.

\section{Analysis and Performance Discussion}

There are three important points to be made, which we will verify in Section 4:

1. Observe that, the lower triangle part of matrix $\mathbf{A}_{k}$ is composed of $\mathbf{x}_{k}^{(f)}$, while the upper part is composed of $\mathbf{x}_{k-1}^{(f)}$. Since, $\mathbf{x}_{k}^{(f)}$ and $\mathbf{x}_{k-1}^{(f)}$ are last $v-1$ samples of $\mathbf{x}_{k}$ and $\mathbf{x}_{k-1}$, respectively, we can say that in the block adaptive channel estimation scheme, the channel estimate $\hat{\mathbf{h}}_{k}$ is based on the data pair $\left(\hat{\mathbf{x}}_{k}, \hat{\mathbf{x}}_{k-1}\right)$. The data pair $\left(\hat{\mathbf{x}}_{k}, \hat{\mathbf{x}}_{k-1}\right)$ is obtained from the channel pair $\left(\hat{\mathbf{h}}_{k-1}, \hat{\mathbf{h}}_{k-2}\right)$. Moreover, $\hat{\mathbf{h}}_{k-1}$ and $\hat{\mathbf{h}}_{k-2}$ are achieved through the corresponding data pairs $\left(\hat{\mathbf{x}}_{k-1}, \hat{\mathbf{x}}_{k-2}\right)$ and $\left(\hat{\mathbf{x}}_{k-2}, \hat{\mathbf{x}}_{k-3}\right)$, respectively. In view of this, we see that for reliable channel estimation the channel should remain quasi-static during four successive OFDM symbols. Consequently, the scheme 
Table 1. CP based Exponentially Weighted Block RLS Channel Estimation Algorithm

Input: $\mathbf{y}_{k}^{(f)}$ and $\mathbf{Y}_{k}$

Selecting parameters: $\mu_{1}$ and $\mu_{2}$

Initialization: $k=0$, an initial training process is used to initialize $\hat{\mathbf{h}}_{0}$ and $\overline{\boldsymbol{\Phi}}_{0}$.

Computation: $k=1,2,3, \cdots$

1. $\hat{\mathcal{H}}_{i, k-1}=\sum_{l=0}^{v} \hat{h}_{l, k-1} e^{-j(2 \pi i l) / m}$, $\hat{W}_{i, k-1}=1 / \hat{\mathcal{H}}_{i, k-1}, i=0, \ldots, m-1$

2. $\hat{X}_{i, k}=Y_{i, k} \hat{W}_{i, k-1}, i=0, \ldots, m-1$

3. $\bar{x}_{i, k}=(1 / \sqrt{m}) \sum_{l=0}^{m-1} q\left(\hat{X}_{l, k}\right) e^{j(2 \pi i l) / m}$, $i=m-v, \ldots, m-1$

4. $\overline{\mathbf{\Phi}}_{k}=\mu_{1} \overline{\boldsymbol{\Phi}}_{k-1}+\sum_{l=1}^{v} \mu_{2}^{l-1} \overline{\mathbf{u}}_{l, k} \overline{\mathbf{u}}_{l, k}^{H}$, $\overline{\mathbf{z}}_{k}=\mu_{1} \overline{\mathbf{z}}_{k-1}+\sum_{l=1}^{v} \mu_{2}^{l-1} \overline{\mathbf{u}}_{l, k} y_{-l, k}$,

5. $\hat{\mathbf{h}}_{k}=\overline{\boldsymbol{\Phi}}_{k}^{-1} \overline{\mathbf{z}}_{k}$,

is suitable to track slowly time varying channels. Towards improving tracking performance of the scheme, the diagonal matrix $\Lambda_{v}$ consisting of weighting factors $\mu_{2}^{l-1}, l=1,2,3, \cdots, v$, is seen to put more emphasis on the data from the first element and less attention to the data from the second element of the data pairs $\left(\hat{\mathbf{x}}_{k}, \hat{\mathbf{x}}_{k-1}\right),\left(\hat{\mathbf{x}}_{k-1}, \hat{\mathbf{x}}_{k-2}\right)$ and $\left(\hat{\mathbf{x}}_{k-2}, \hat{\mathbf{x}}_{k-3}\right)$. However, since the loss of available information is related to both the elements of data pairs, therefore the channel estimation scheme is more likely to have poor performance with reduced values of $\mu_{2}$ and thus corresponding exponential forgetting weightings.

2. Symbol recovery through frequency domain one tap equalization is not assured if the channel has nulls on (or close to) some subcarriers. Due to this limitation, performance of the decision directed block RLS channel estimation algorithm may therefore not be satisfactory in channels suffering from deep channel fades.

3. The modulation alphabet complexity determines the number of bits that can be transmitted with one modulated symbol. The more complex the alphabet, the narrower the gap between the symbol decision space and higher the probability of error in detecting the signal. Since the block RLS algorithm relies on the finite alphabet (FA) property of source symbols, high performance degradation is expected as the constellation size increases.
Table 2. Static FIR Channel Models

\begin{tabular}{c|c|c|c|c}
\hline \hline tap \# & Chnl. A & Chnl. B & Chnl. C & Chnl. D \\
\hline 0 & 0.3122 & 0.5312 & 0.4122 & 0.2000 \\
\hline 1 & 0.0501 & 0.5010 & 0.0501 & 0.0000 \\
\hline 2 & 0.2056 & 0.2056 & 0.3000 & 0.0000 \\
\hline 3 & 0.2754 & 0.4754 & 0.2000 & 0.0000 \\
\hline 4 & 0.1567 & 0.8000 & 0.2567 & 0.2000 \\
\hline \hline
\end{tabular}

\section{Simulation Results}

In this section we present simulation examples, which were carried out with $m=52, v=4, \mu_{1}=0.7$, $\mu_{2}=1, \mathrm{SNR}=25 \mathrm{~dB}$ and 4-QAM, unless otherwise stated. The transmit power of all used subchannels is equal and $\sigma_{i}^{2}=\sigma^{2}$. The performance is evaluated by averaged meansquare-error (MSE) per subchannel err $=\sum_{i \in U} \| X_{i}-$ $\hat{X}_{i}|| /|U|$, where $U$ is the set of indexes corresponding to the $U$ used subchannels and $|U|$ is the number of all the used subchannels [4]. The fours static finite impulse response (FIR) channels used in simulation examples are listed in Table 2, whereas, corresponding frequency responses are shown in Fig. 2. Also, only the first OFDM symbol was sent as pure training sequence to identify the initial channel $\mathbf{h}_{0}$ for fast convergence and simulation results were averaged over 300 Monte Carlo runs.

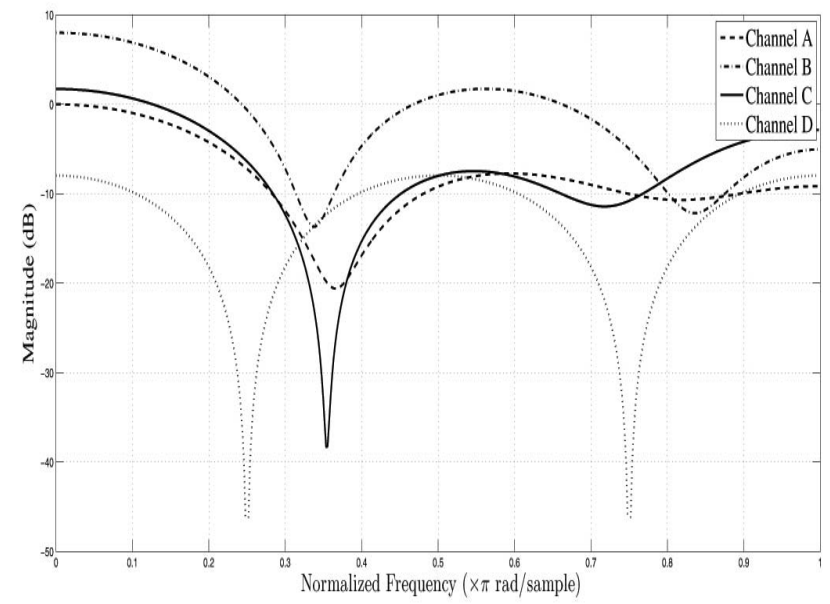

Figure 2. Frequency responses (magnitude) of FIR channels.

Example 1: The goal of this example is to test the performance of the algorithm to converge and track abrupt 


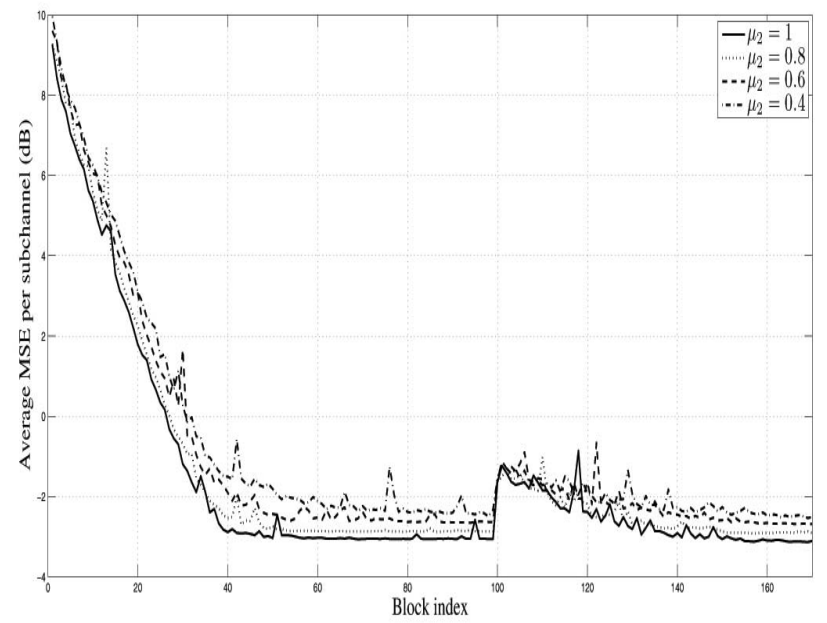

Figure 3. Average MSE per subchannel corresponding to three choices of parameter $\mu_{2}$.

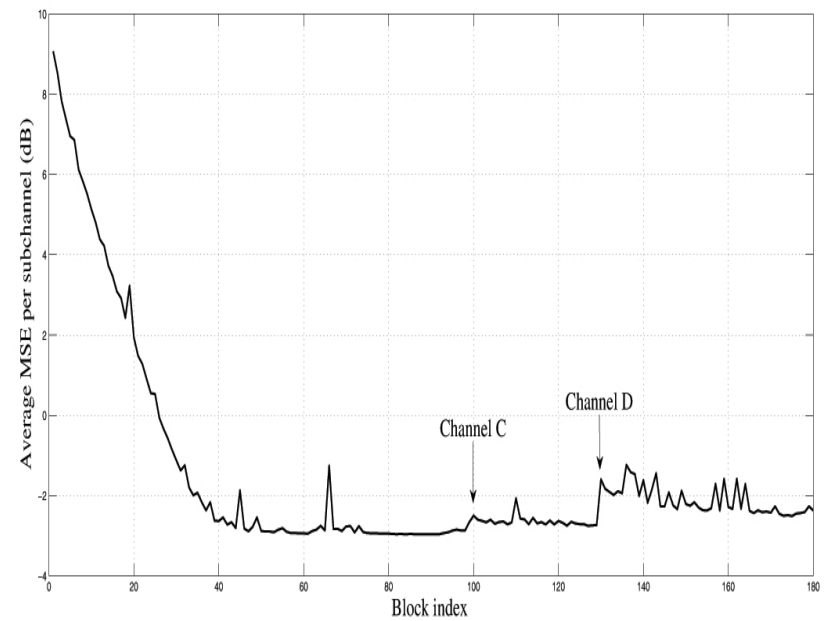

Figure 4. Average MSE per subchannel curve when tracking Channel $C$ and $D$ with spectral nulls.

changes with varying exponential forgetting weightings by varying $\mu_{2}$. Fig. 3 shows typical performance curves of the algorithm for 4 values of $\mu_{2}$. The channel is having impulse response of Channel $A$, which remains unchanged for the first 100 data blocks. At data block 101 we switch to Chan$n e l B$. It can be seen from Fig. 3 that the algorithm converges in all cases and is able to track the channel variation. No practical difference in tracking is seen with varying $\mu_{2}$.

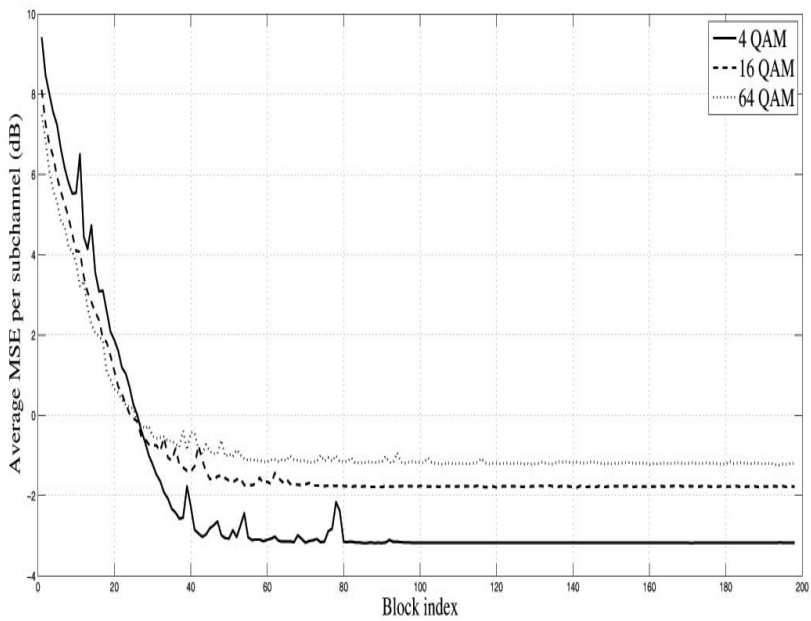

Figure 5. Average MSE per subchannel performance with three choices of QAM constellation size.

The convergence time and steady state performance error increases with decreasing $\mu_{2}$ (i.e., with smaller values of exponential forgetting weightings) and is smallest for $\mu_{2}=1$. We remark here that $\mu_{2}=1$ means that $\Lambda_{v}=\mathbf{I}$.

Example 2: Here we examine performance of the adaptive algorithm in presence of channel nulls. Fig. 4 shows variation in MSE, as we switch from Channel $\mathrm{A}$ to $\mathrm{C}$ at data block 101 and from Channel C to D at data block 131. Notice that Channel C carries one spectral null, whereas, Channel D is more severe and has two deep nulls across its spectrum. We can see that the algorithm performs poorly due to noise enhancement caused by increasing number of spectral nulls.

Example 3: In this last simulation example, we repeat Example 1 with Channel A only and varying constellation size. Corresponding simulation results in Fig. 5 show that the algorithm takes same number of data blocks to converge. However, as expected, the performance of the algorithm degrades with increasing constellation size. The algorithm is therefore not suitable for modulation schemes with large constellations.

\section{Conclusion}

In this paper, performance of $\mathrm{CP}$ based exponentially weighted block RLS channel estimation algorithm was analyzed. The algorithm was tested for time varying environments with varying exponential forgetting weightings, number of channel nulls and constellation size. The algorithm demonstrated slow convergence and increased error during 
steady state performance with the decrease in exponential forgetting weightings. The algorithm also showed performance degradation with the increase in channel nulls and constellation size.

In conclusion, we emphasize that tracking performance of the algorithm does not depend on exponential forgetting weightings, and to improve the algorithm convergence and steady performance $\mu_{2}=1$ should be pursued as an option. With $\mu_{2}=1$, the algorithm degenerates to the un-weighted block RLS algorithm in [4]. The only difference (between exponentially weighted and un-weighted block RLS schemes) appears in the equalization stage, and since ZF equalization in OFDM in general outperforms the MMSE equalization (although the latter offers lower MSE) [7], for enhanced capability, the un-weighted block RLS algorithm should be pursued with ZF equalization. To overcome the problem of channel nulls, in absence of errorcontrol coding, power loading is recommended to optimize the error rate performance. Also, due to poor performance with larger constellations, the scheme may not be suitable for rate adaptation. Therefore, its implementation be limited with low constellation sizes.

\section{References}

[1] IEEE Part II: Wireless LAN Medium Access Control (MAC) and Physical Layer (PHY) Specifications: High-Speed Physical Layer in $5 \mathrm{GHz}$ Band. IEEE Sts. 802.11a-1999, 1999.

[2] K. V. Acker, G. Leus, M. Moonen, O. van de Wiel, and T. Pollet. Per tone equalization for DMT-based systems. IEEE Trans. Commun., 49(1):109-119, Jan. 2001.

[3] J. S. Chow, J. C. Tu, and J. M. Cioffi. A discrete multitone transceiver system for HDSL application. IEEE J. Select. Areas Commun., 9:895-908, Aug. 1991.

[4] X. Wang and K. J. R. Liu. Adaptive channel estimation using cyclic prefix in multicarrier modulation system. IEEE Commun. Lett., 3(10):291-293, Oct 1999.

[5] X. Wang and K. J. R. Liu. Performance analysis for adaptive channel estimation exploiting cyclic prefix in multicarrier modulation systems. IEEE Trans. Commun., 51(1):94-103, Jan. 2003.

[6] Z. Wang and G. B. Giannakis. Wireless multicarrier communications where Fourier meets Shannon. IEEE Signal Processing Magazine, pages 29-48, May 2000.

[7] Z. Wang, X. Ma, and G. B. Giannakis. OFDM or singlecarrier block transmissions? IEEE Trans. Commun., 52:380394, Mar. 2004. 